\title{
Size Dependent Optical Nonlinearities of InP/ZnS Core/Shell Quantum Dot
}

\author{
M. Elamathi' ${ }^{1}$ A. John Peter ${ }^{2, *}$ \\ ${ }^{1}$ Department of Physics, Saiva Bhanu Kshatriya College, Aruppukkottai - 626 101, Tamilnadu, India. \\ ${ }^{2} P G$ and Research Deptartment of Physics, Government Arts College, Melur, Madurai - 625 106, Tamilnadu, India.
}

\section{ART ICLE DETAILS}

\section{Article history:}

Received 21 March 2018

Accepted 29 March 2018

Available online 06 April 2018

\section{Keywords:}

Exciton

Quantum Dot

Optical Gain

\begin{abstract}
A B S T R A C T
In the present work, the size dependent electronic and optical nonlinearities of InP/ZnS core/shell quantum dot are investigated. The impurity states in $\mathrm{InP} / \mathrm{ZnS}$ core/shell quantum dot are studied taking into account the geometrical confinement effect. The energy eigen values and the exciton binding energies are found using variational formulism with two variational parameters. Some nonlinear optical properties are concentrated. The exciton binding energies, optical band gap and the optical gain, in $\mathrm{InP} / \mathrm{ZnS}$ quantum dot, are obtained. The optical gain as a function of photon energy is found using compact density approach. The optical gain in any reduced dimensional semiconductor system proposes an essential information about the heterostructure. The larger exciton binding energy of InP/ZnS quantum dot implies the wider size tuning within the strong confinement region. It has been observed that InP/ZnS core /shell quantum dots are safer, low toxicity and environmental friendly and is considered to be an alternative to $\mathrm{CdSe} / \mathrm{ZnS}$ based quantum dots which cannot be used for bio-medical applications.
\end{abstract}

\section{Introduction}

Quantum dots (QD) with the exotic physical and chemical properties show great interests in the optical applications such as laser diodes, sensors and light emitting diodes (LED). Size quantization in all the directions are involved in any quantum dot which picks up the name, artificial atom. Geometrical size tunable energy band gaps, virtuous photostability and high efficiency are some superior properties which are required for fabricating the best QD-LEDs. InP based LEDs have proven easy to synthesize having superior electro-optical properties [1]. Size tunable absorption and emission spectra using InP materials have been performed in the visible and near infrared wavelength ranges [2]. Biorelated application of InP quantum dots as efficient energy donors for resonance energy transfer has been reported earlier [3]. Size dependent electronic properties of InP quantum dots based on density functional theory have been investigated and a reliable analytical equation has been described with the change of energy band gap as a function of size [4]. Colloidal dispersions of InP/ZnS nanoparticles have been prepared using a single-step heating-up method relying at low temperature and the optical properties have been studied [5]. The third-order optical nonlinearities of high-quality colloidal InP/ZnS core-shell quantum dots using Z-scan technique with femtosecond pulses have been studied and suggested that this core/shell material could be applied for a variety of potential applications [6]. Considering the environmental facts, Cadmium free LEDs such as InP based LEDs are preferred for focusing green synthesis leading to low cost preparation. Especially, InP/ZnS core/shell QD-LEDs exhibit more than 80\% efficiency [7]. The core/shell quantum dot heterostructures are given attention due to the encapsulation in many systems which can employ effectively as active materials in a lot of optical applications. Size dependent tunable effective band gap of the above systems can be used for fabricating novel optical devices within the control over emission wavelength especially in the fibre optical communication with the minimum loss.

\section{Theoretical Formulism}

InP is taken as shell material with the inner radius, $R_{\mathrm{c}}$ and the dielectric constant, $\varepsilon_{c}$, embedded on an outer core shell, $\mathrm{ZnS}$, material with the outer radius, $R_{\mathrm{s}}$ and the dielectric constant $\left(\varepsilon_{\mathrm{s}}\right)$. The two particle Hamiltonian, within the single band effective mass approximation, is given by

$$
H=\sum_{i=e, h[}\left[-\frac{\hbar^{2}}{2 m_{i}^{*}} \nabla^{2}+V_{i}\left(r_{i}\right)\right]-U\left(r_{e}, r_{h}\right)
$$

where i=e,h refer the carrier choice, $V_{i}\left(r_{i}\right)$ is the confinement potential of each section. The first term represents the kinetic energy operator. $U\left(r_{e}, r_{h}\right)$ is the potential energy term is given by

$$
U\left(r_{e}, r_{h}\right)=-\frac{e^{2}}{\varepsilon\left|\vec{r}_{e}-\vec{r}_{h}\right|}
$$

The dielectric constant of the inner core material is greater than the shell material. It follows as

$$
\varepsilon\left(r_{e}, r_{h}\right)= \begin{cases}\varepsilon_{C} & \\ \frac{\varepsilon_{S}\left(r^{3}-R_{S}^{3}\right)+\varepsilon_{S}\left(R_{S}^{3}-R_{C}^{3}\right)+\varepsilon_{C} R_{C}^{3}}{R_{S}^{3}} & r<R_{C} \\ & r>R_{S}\end{cases}
$$

where $\varepsilon_{\mathrm{c}}$ and $\varepsilon_{\mathrm{s}}$ are the dielectric constants of core and the shell materials respectively.

The barrier potentials, $V_{e(h)}(z)$, related to the band offsets in the $\mathrm{InP} / \mathrm{ZnS}$ structure are given by

$$
V_{e(h)}(z)= \begin{cases}V_{e(h)}^{0} & r<R_{C} \\ V_{e(h)}^{0} & r>R_{S}\end{cases}
$$

where $V_{e(h)}$ is the barrier height of conduction (valence) band. The lowest binding energies, the exciton binding energies and the related wave functions are the solution of Schrödinger equation. The single particle Schrödinger wave equation of an exciton can be written as 


$$
H \Psi\left(r_{e}, r_{h}\right)=E \Psi\left(r_{e}, r_{h}\right)
$$

The envelope function is the multiplication of radial wave functions and angular parts of electron and hole wave functions. The eigen state of the wave function can be written in the form of

$$
\psi_{n l m}(r, \theta, \phi)=R_{n l}(r) Y_{l m}(\theta, \phi)
$$

with $R_{n l}(r)$ and $Y_{l m}$ are the radial and the spherical harmonic wave functions respectively.

The assumed single particle wave function of the exciton is followed as

$$
\Psi(r)=N \psi_{n l m}(r, \theta, \phi) \exp (-\lambda r)
$$

where $\psi_{n l m}(r, \theta, \phi)$ is the ground state solution of the Schrödinger equation for the electron (hole) in the absence of the Coulomb interaction as given in Eq.(7) and $\mathrm{N}$ is the normalization constant. $\lambda$ is the variational parameter. The assumed wave functions of exciton must satisfy the continuity condition at the boundaries of inner and outer materials of hetero-structure. This can be helped to determine the respective binding energies of exciton with the inclusion of spatial confinement effects. Thus, the variational parameter is employed to obtain the exciton ground state energy by minimizing Eq.(1)

$$
E=\min _{\lambda} \frac{\langle\Psi|H| \Psi\rangle}{\langle\Psi \mid \Psi\rangle}
$$

The exciton binding energy of the system is given as

$$
E_{b}=E_{e}+E_{h}-\langle H(\lambda)\rangle_{\min } .
$$

with $E_{e}$ and $E_{h}$ are the energy eigenvalues of the single electron and hole without the interaction term between them. $\langle H(\lambda)\rangle_{\min }$ is calculated by minimizing the (Eq.(1)) in respect of the variational parameters which are given in Eq.(7). The Schrödinger equation is solved variationally by finding $\langle H(\lambda)\rangle_{\min }$ and the binding energy of the exciton in a core/shell is given by the difference between the energy with and without the Coulomb interaction.

The expression for the laser induced optical gain for the interband optical transitions is given by [8]

$$
\begin{aligned}
\alpha\left(\omega, \alpha_{0}\right)= & \frac{\omega \mu c e^{2}}{\eta}\left|M_{f i}\right|^{2} \frac{m^{*} k_{B} T}{R \pi \hbar^{2}} \times \ln \left[\frac{1+\exp \left[\left(E_{F}-E_{i}\right) / k_{B} T\right]}{1+\exp \left[\left(E_{F}-E_{f}\right) / k_{B} T\right]}\right] \times \\
& \frac{\hbar / \Gamma_{i n}}{\left(E_{f}-E_{i}+M \omega^{2} \alpha_{0}^{2} / 2-\hbar \omega\right)^{2}+\left(\hbar / \Gamma_{i n}\right)^{2}}
\end{aligned}
$$

where e is the absolute value of the electron charge, $\mu$ is the permeability of the InP/ZnS quantum dot material, refractive index, $\mathrm{c}$ is the speed of light in free space, $\Gamma_{\text {in }}$ is the relaxation time, $\mathrm{E}_{\mathrm{f}(\mathrm{i})}$ is the final and initial state energy, $\omega$ is the angular frequency of optical radiation, $\mathrm{k}_{\mathrm{B}}$ is the Boltzmann constant, $\mathrm{T}$ is the temperature, $\mathrm{E}_{\mathrm{F}}$ represents the Fermi energy and $M_{f i}$ is the matrix element given by the expression as

$$
M_{f i}=\int \psi_{f}^{*}(r) r \psi_{i} d r
$$

where $\psi_{f}$ and $\psi_{r}$ refer the initial and final state wave functions of the optical transition. For this purpose, we use the single band effective mass approximation and expand the electron wave function in an appropriate set of orthonormal functions. Then, by using the density matrix approach, within a two-level system approach, the explicit expressions for the nonlinear optical properties computed in a saturation limit.

\section{Results and Discussion}

The binding energies due to heavy hole exciton are found employing variational formulism as the heavy holes are often used in experimental sides. The electronic properties are obtained using variational approach whereas the optical properties are dealt using the compact density matrix method with the iteration process. In Fig. 1, the variation of optical gain as a function of photon energy is shown for the InP/ZnS core /shell quantum dot with the constant electron density. The dot radius is taken as $40 \AA$ and the electron density is taken as $2 \times 10^{6} \mathrm{~cm}^{-1}$. The optical gain in any low dimensional semiconductor offers an important information about the heterostructure [9]. The corresponding lasing wavelength is observed to be $1.551 \mu \mathrm{m}$ which is the range of wavelength in telecommunication network for the optical window system to minimize the loss [10]. Generally, the fiber-optic communication is mainly conducted in the wavelength region $(1.3 \mu \mathrm{m}-1.55 \mu \mathrm{m})$ in which the optical fibers have minimum transmission loss. Thus, the desired output wavelength for fibre optical communication can be achieved in the present system with $\mathrm{InP} / \mathrm{ZnS}$ heterostructure core/shell quantum dot.

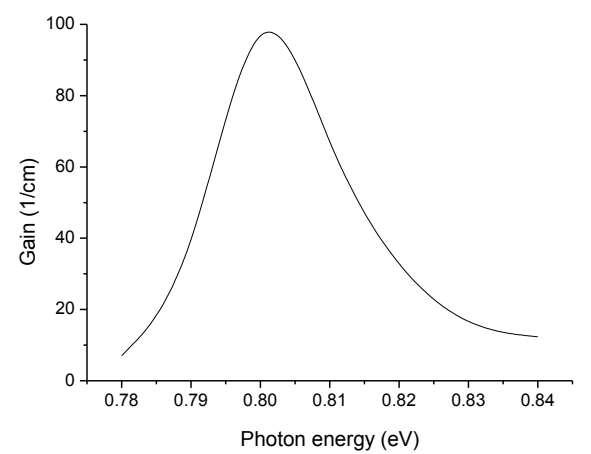

Fig. 1 Variation of optical gain with the photon energy in the InP/ZnS core/shell quantum dot

\section{Conclusion}

The fiber-optic communication is mainly conducted in the wavelength region $(1.3 \mu \mathrm{m}-1.55 \mu \mathrm{m})$ in which the optical fibers have minimum transmission loss. Thus, the desired output wavelength for fibre optical communication can be achieved in the present system with $\mathrm{InP} / \mathrm{ZnS}$ heterostructure core/shell quantum dot.

\section{References}

[1] T.R. Kuo, S.T. Hung, Y.T. Lin, T.L. Chou, M.C. Kuo, Y.P. Kuo, C.C. Chen, Green synthesis of InP/ZnS Core/shell quantum dots for application in heavy-metalfree light-emitting diodes, Nanoscale Res. Lett. 12 (2017) 537-544.

[2] H. Chibli, L. Carlini, S. Park, N.M. Dimitrijevic, J.L. Nadeau, Cytotoxicity of InP/ZnS quantum dots related to reactive oxygen species generation, Nanoscale 3 (2011) 2552-2559.

[3] A. Thomas, P.V. Nair, K. George Thomas, InP quantum dots: an environmentally friendly material with resonance energy transfer requisites, J. Phys. Chem. C 118 (2014) 3838-3845.

[4] E. Cho, H. Jang, J. Lee, E. Jang, Modeling on the size dependent properties of InP quantum dots: a hybrid functional study, Nanotech. 24 (2013) 215201-215206.

[5] J.S. Arias-Cerón, M.P. González-Araoz, A. Bautista-Hernández, J.L. Sánchez Ramírez, J.F. Herrera-Pérez, J.G. Mendoza-Álvarez, Semiconductor nanocrystals of InP@ZnS: synthesis and characterization, Superficies y Vacío 25 (2012) 134-138.

[6] Y. Wang, X. Yang, T.C. He, Y. Gao, H.V. Demir, X.W. Sun, H.D. Sun, Near resonant and nonresonant third order optical nonlinearities of colloidal $\mathrm{InP} / \mathrm{ZnS}$ quantum dots, Appl. Phys. Lett.102 (2013) 021917-021921.

[7] S. Kim, T. Kim, M. Kang, S.K. Kwak, T.W. Yoo, L.S. Park, et al., Highly luminescent InP/GaP/ZnS Nanocrystals and their application to white light-emitting diodes, J. Am. Chem. Soc.134 (2012) 3804-3809.

[8] M. Marinescu, M. Gavrila, First iteration within the high-frequency Floquet theory of laser-atom interactions, Phys. Rev. A 53 (1995) 2513-2521.

[9] S. Saravanan, A. John Peter, Chang Woo Lee, Laser field induced optical gain in a group III-V quantum wire, Eur. Phys. J. D 70 (2016) 165-170.

[10] D. Fuster, L. González, Y. González, J. Martínez-Pastor, T. Ben, A. Ponce, S.I. Molina, Emission wavelength engineering of InAs/InP(001) quantum wires, Eur. Phys. J. B. 40 (2004) 433-437.

\footnotetext{
About the Conference: "International Conference on Nanomaterials (ICAN) - 2018" has been organized by Dr. G. Ramalingam, ICAN2018 Organizing Secretary, Assistant Professor, Department of Nanoscience and Technology, Alagappa University, Karaikudi, TN, India at his designated venue on $26^{\text {th }} \& 27^{\text {th }}$ February 2018.
} 